\title{
NILPOTENCY OF DERIVATIVES ON AN IDEAL
}

\author{
L. O. CHUNG AND JIANG LUH
}

\begin{abstract}
Let $R$ be a prime ring and $\partial$ a derivation of $R$. It is shown that if $\partial^{m} I=(0)$ for some nonzero ideal $I$ in $R$, then $\partial^{m} R=(0)$.
\end{abstract}

1. Introduction. Let $R$ be a prime ring and $\partial$ a nilpotent derivation of $R$ (i.e. $\partial$ is a derivation of $R$ with $\partial^{m} R=(0)$ for some positive integer $m$ ). Recently [1,2], we have shown that the nilpotency of $\partial$ must be odd if $R$ is not of characteristic 2, but must be a power of 2 if $R$ is of characteristic 2. It is also known (see [4,p. 7]) that if $\partial I=(0)$ for some nonzero one-sided ideal $I$ in $R$, then $\partial R=(0)$. A natural question therefore arises: If $\partial$ is nilpotent on a nonzero one-sided ideal in $R$, is $\partial$ necessarily nilpotent on $R$ ? If so, should the nilpotency of $\partial$ on $I$ equal the nilpotency of $\partial$ on $R$ ? In answering these questions we shall first show a rather easy result: if $\partial^{m} I=(0)$ then $\partial^{2 m-1} R=(0)$. Then using some techniques in [1] we shall prove our main result.

THEOREM. Let $R$ be a prime ring and I a nonzero two-sided ideal in $R$. Suppose $\partial$ is a derivation of $R$ such that $\partial^{m} I=(0)$. Then $\partial^{m} R=(0)$.

This result can be used to improve the main result in [3] by replacing $R$ by any nonzero ideal in $R$. There we study algebraic derivations (i.e. derivations satisfying an algebraic polynomial $f(x))$ and prove the following sharpened version of the well-known result of V. K. Kharchenko: An algebraic derivation of a prime ring of characteristic zero is an inner derivation in the generalized quotient ring $Q(R)$ of $R$. Moreover, it is induced by an element $a$ in $Q(R)$ of the form $a=\sum_{i=1}^{n} \alpha_{i} e_{i}+b$, where $b$ is nilpotent, $\left\{e_{1}, e_{2}, \ldots, e_{n}\right\}$ is a set of orthogonal idempotents, $b e_{i}=e_{i} b$, and the $\alpha_{i}$ 's are certain roots of $f(x)$ in the center of $Q(R)$.

A similar result of our present main theorem on Lie ideals of prime rings is also true. The latter result will appear in the dissertation of David W. Jensen of North Carolina State University.

Received by the editors June 23, 1983.

1980 Mathematics Subject Classification. Primary 16A72; Secondary 16A12.

Key words and phrases. Derivations, prime rings, nilpotency.

(C)1984 American Mathematical Society $0002-9939 / 84 \$ 1.00+\$ .25$ per page 
2. Preliminary results. We shall require the following results.

Proposition 1. Let $0<i \leqslant t$ be integers and

$$
f(t, i)=\left|\begin{array}{cccccc}
0 & 0 & \ldots & 0 & \left(\begin{array}{l}
t \\
0
\end{array}\right) & \left(\begin{array}{l}
t \\
1
\end{array}\right) \\
0 & 0 & \cdots & \left(\begin{array}{c}
t \\
0
\end{array}\right) & \left(\begin{array}{l}
t \\
1
\end{array}\right) & \left(\begin{array}{l}
t \\
2
\end{array}\right) \\
\cdot & \cdot & \ldots & \cdot & \cdot & \cdot \\
\left(\begin{array}{l}
t \\
0
\end{array}\right) & \left(\begin{array}{l}
t \\
1
\end{array}\right) & \cdots & \left(\begin{array}{c}
t \\
i-3
\end{array}\right) & \left(\begin{array}{c}
t \\
i-2
\end{array}\right) & \left(\begin{array}{c}
t \\
i-1
\end{array}\right) \\
\left(\begin{array}{l}
t \\
1
\end{array}\right) & \left(\begin{array}{l}
t \\
2
\end{array}\right) & \ldots & \left(\begin{array}{c}
t \\
i-2
\end{array}\right) & \left(\begin{array}{c}
t \\
i-1
\end{array}\right) & \left(\begin{array}{l}
t \\
i
\end{array}\right)
\end{array}\right|
$$

Then

$$
f(t, i)=(-1)^{i(i-1) / 2}\left(\begin{array}{c}
t+i-1 \\
i
\end{array}\right)
$$

(II) if $p$ is an odd prime number, $n<t \leqslant 2 n$ are integers, $n=\sum_{j=0}^{N} \alpha_{j} p^{j}$, where $\alpha_{0}$, $\alpha_{N} \neq 0$ and $0 \leqslant \alpha_{j}<(p-1) / 2$ for $j=0,1,2, \ldots, N$, then there exists $i$ such that $2 n-t+1 \leqslant i \leqslant t-1$ and $f(t, i) \neq 0(\bmod p)$.

The proof can be found in [1].

Proposition 2. Let $R$ be a prime ring and I a nonzero right ideal in $R$. If $\partial$ is a derivation of $R$ such that $\partial^{m} I=(0)$ for some positive integer $m$, then $\partial^{2 m-1} R=(0)$.

Proof. Note that $J=I+\partial I+\cdots+\partial^{m-1} I$ is also a right ideal in $R, \partial J \subseteq J$ and $\partial^{m} J=(0)$. Thus without loss of generality we may assume $\partial I \subseteq I$. Let $a \in I$ and $x \in R$. By Leibniz' rule, $0=\partial^{m}\left(\partial^{m-1} a x\right)=\partial^{m-1} a \partial^{m} x$. Using this and Leibniz' rule again, we obtain $0=\partial^{m}\left(\partial^{m-2} a \partial x\right)=\partial^{m-2} a \partial^{m+1} x$. Continuing this argument we finally reach the identity $0=a \partial^{2 m-1} x$ for all $a \in I$ and $x \in R$. Hence, by the primeness of $R, \partial^{2 m-1} x=0$ for all $x \in R$.

3. Proof of Theorem. As in the proof of Proposition 2, we may assume $\partial I \subseteq I$. Also we may assume $m$ is the nilpotency of $\partial$ on $I$, i.e. $\partial^{m} I=(0)$ but $\partial^{m-1} I \neq(0)$.

Suppose $R$ is of characteristic 2 . Then since $I$ itself is a prime ring of characteristic $2, m=2^{k}$ for some positive integer $k$, and $\delta=\partial^{m}$ is also a derivation of $I$ with $\delta I=(0)$. Consequently, $\delta R=(0)$ or $\partial^{m} R=(0)$.

Thus throughout the balance of the proof we assume the characteristic $p$ of $R$ is either zero or an odd prime.

Suppose, to the contrary, the theorem were false. Let $n$ be the least positive integer with respect to the property: There is a derivation $\partial$ of $R$ such that $\partial^{2 n+1} R=(0)$, $\partial^{2 n} R \neq(0)$ but $\partial^{2 n} I=(0)$.

Claim 1. If $b \in R$ and $\left(\partial^{n} I\right) b=(0)$, then $b=0$. For any $a \in I$ and $x \in R$, $0=\partial^{n}\left(x \partial^{n-1} a\right) b=\partial^{n} x \partial^{n-1} a b$. Using this and Leibniz' rule, we get $0=$ $\partial^{n}\left(\partial x \partial^{n-2} a\right) b=\partial^{n+1} x \partial^{n-2} a b$. Continuing this process, we finally get $0=\partial^{2 n-1} x a b$ for all $a \in I$ and $x \in R$. By the primeness of $R, b=0$. 
Claim 2. If $\left(\partial^{t} I\right) b=0$ where $b \in R$ and $n \leqslant t<2 n$, then $\left(\partial^{t} R\right)\left(\partial^{t-1} I\right) b=(0)$. This is true since, for $a \in I$ and $x \in R, 0=\partial^{t}\left(x \partial^{t-1} a\right) b=\left(\partial^{t} x\right)\left(\partial^{t-1} a\right) b$.

Claim 3. If the characteristic $p$ is not zero, then $n=\sum_{j=0}^{N} \alpha_{j} p^{j}$, where $0 \leqslant \alpha_{j}<$ $(p-1) / 2$ are integers for $j=0,1,2, \ldots, N$ and $\alpha_{N} \neq 0$. We write $2 n=\sum_{j=0}^{N} \beta_{j} p^{j}$ where $0 \leqslant \beta_{j} \leqslant p$ are integers for $j=0,1,2, \ldots, N$ and $\beta_{N} \neq 0$. What we need to show is that $\beta_{j}$ is even for each $j$. First, suppose $\beta_{N}$ were odd. Then $\left(\beta_{N}+1\right) p^{N}$ would be even and $>2 n$ and, moreover, $\delta=\partial^{p^{N}}$ would be a derivation of $R$ with $\delta^{\beta_{N}+1} R=(0)$. Since the nilpotency of $\delta$ is necessarily odd, it would imply $\delta^{\beta_{N}} R=(0)$ or $\delta^{\beta_{N} p^{N}} R=(0)$ and, hence, $\partial^{2 n} R=(0)$, a contradiction. Thus $\beta_{N}$ is even. Now suppose for some $j, \beta_{j}$ were odd. We choose $j$ to be the largest one, i.e. $\beta_{j+1}$, $\beta_{j+2}, \ldots, \beta_{N}$ are even and $\beta_{j}$ is odd, where $0 \leqslant j<N$. Then

$$
2 n<\left(\beta_{j}+1\right) p^{j}+\beta_{j+1} p^{j+1}+\cdots+\beta_{N} p^{N}=\lambda p^{j},
$$

where $\lambda=\left(\beta_{j}+1\right)+\beta_{j+1} p+\cdots+\beta_{N} p^{N-j}$. Note that $\delta=p^{j}$ is a derivation of $R$ with $\delta^{\lambda} R=(0)$. Since $\lambda$ is even, $\delta^{\lambda-1} R=(0)$ which implies $\partial^{2 n} R=(0)$, again a contradiction. Hence all $\beta_{j}$ 's are even, as we desired.

Claim 4. $\alpha_{0} \neq 0$. Suppose $\alpha_{0}=0$. Then $n=\lambda p$ where $\lambda=\sum_{j=1}^{N} \alpha_{j} p^{j-1}$, and $\delta=\partial^{p}$ is a derivation of $R$ with $\delta^{2 \lambda+1} R=\partial^{2 n+p} R=(0), \delta^{2 \lambda} R=\partial^{2 n} R \neq(0)$ and $\delta^{2 \lambda} I=\partial^{2 n} I=(0)$, which contradicts the minimality of $n$ since $\lambda<n$. Therefore, $\alpha_{0} \neq 0$.

Claim 5. Let $a \in R$ and $t$ be an integer, $n<t \leqslant 2 n$. Suppose $\left(\partial^{t} R\right) a=(0)$. Then $\left(\partial^{t-1} I\right)\left(\partial^{t-1} R\right) a=(0)$. Indeed, for $x \in I, y \in R$ and $i=0,1,2, \ldots, t-2$, $\partial^{t}\left(\partial^{i} x \partial^{t-2-i} y\right) a=0$ yields

$$
\left(\left(\begin{array}{l}
t \\
0
\end{array}\right) \partial^{t+i} x \partial^{t-2-i} y+\left(\begin{array}{l}
t \\
1
\end{array}\right) \partial^{t+i-1} x \partial^{t-1-i} y+\cdots+\left(\begin{array}{c}
t \\
i+1
\end{array}\right) \partial^{t-1} x \partial^{t-1} y\right) a=0 .
$$

These $t-1$ equations can be expressed in matrix form by noting that $\partial^{2 t-2} x=0$ :

$$
\left[\begin{array}{cccccc}
0 & 0 & \cdots & 0 & \left(\begin{array}{l}
t \\
0
\end{array}\right) & \left(\begin{array}{l}
t \\
1
\end{array}\right) \\
0 & 0 & \cdots & \left(\begin{array}{c}
t \\
0
\end{array}\right) & \left(\begin{array}{l}
t \\
1
\end{array}\right) & \left(\begin{array}{l}
t \\
2
\end{array}\right) \\
\cdot \\
\cdot \\
\cdot & \cdots & \cdot & \cdot \\
\left(\begin{array}{l}
t \\
0
\end{array}\right) & \left(\begin{array}{l}
t \\
1
\end{array}\right) & \cdots & \left(\begin{array}{c}
t \\
t-4
\end{array}\right) & \left(\begin{array}{c}
t \\
t-3
\end{array}\right) & \left(\begin{array}{c}
t \\
t-2
\end{array}\right) \\
\left(\begin{array}{l}
t \\
1
\end{array}\right) & \left(\begin{array}{l}
t \\
2
\end{array}\right) & \ldots & \left(\begin{array}{c}
t \\
t-3
\end{array}\right) & \left(\begin{array}{c}
t \\
t-2
\end{array}\right) & \left(\begin{array}{c}
t \\
t-1
\end{array}\right)
\end{array}\right)\left(\begin{array}{c}
\partial^{2 t-3} x \partial y a \\
\partial^{2 t-4} x \partial^{2} y a \\
\vdots \\
\partial^{2 n} x \partial^{2 t-2 n-2} y a \\
\partial^{2 n+1} x \partial^{2 t-2 n-1} y a \\
\vdots \\
\partial^{t-1} x \partial^{t-1} y a
\end{array}\right)=0 .
$$

If the characteristic of $R$ is zero, then, since the determinant of the coefficient matrix is

$$
f(t, t-1)=(-1)^{(t-1)(t-2) / 2}\left(\begin{array}{c}
2 t-2 \\
t-1
\end{array}\right) \neq 0
$$

we obtain $\partial^{t-1} x \partial^{t-1} y a=0$. Now suppose the characteristic of $R$ is not zero. We should note that

$$
\partial^{2 t-3} x \partial y a=\partial^{2 t-4} x \partial^{2} y a=\cdots=\partial^{2 n} x \partial^{2 t-2 n-2} y a=0,
$$


and by Proposition 1(II), there exists $i$ such that $2 n-t+1 \leqslant i \leqslant t-1$ and $f(t, i) \neq 0(\bmod p)$, i.e. there is an upper right corner submatrix of the coefficient matrix which is nonsingular modulo $p$. Hence $\partial^{t-1} x \partial^{t-1} y a=0$.

Claim 6. Let $b \in R$ and $t$ be an integer $n \leqslant t<2 n$. If $\left(\partial^{t} I\right) b=(0)$ then $b=0$. Suppose not. Let $t$ be the smallest positive integer with $\left(\partial^{t} I\right) b=(0)$ for some $b \neq 0$ in $R$. By Claim 1, $t>n$. From Claim 2, $\left(\partial^{t} R\right)\left(\partial^{t-1} I\right) b=(0)$, and by Claim 5 , $\left(\partial^{t-1} I\right)\left(\partial^{t-1} R\right)\left(\partial^{t-1} I\right) b=(0)$. It follows that $\left(\partial^{t-1} R\right)\left(\partial^{t-1} I\right) b=(0)$ by the minimality of $t$. Repeatedly using Claim 5 we finally get $\left(\partial^{n} R\right)\left(\partial^{t-1} I\right) b=(0)$, which by Claim 1 implies $\left(\partial^{t-1} I\right) b=(0)$, a contradiction.

Recall that $m$ denotes the nilpotency of $\partial$ on $I$.

Claim 7. $n+1 \leqslant m<2 n$. Since $\partial^{2 n} I=(0)$ and the nilpotency of $\partial$ on $I$ must be odd, we have $m<2 n . n+1 \leqslant m$ follows from Claim 1 .

Claim $8 . \partial^{m-1} I=(0)$ and hence we reach a contradiction. Indeed $\partial^{m} I=(0)$ yields $\partial^{m-1} I \partial^{m} R=(0)$, which by Claims 6 and 7 implies $\partial^{m-1} I=(0)$. This completes the proof.

EXAMPLE. Let $R$ be the $4 \times 4$ matrix ring over the real field. Let $I=\left\{\left(x_{i j}\right) \in R \mid\right.$ $x_{i j}=0$ for all $\left.i \neq 1\right\}$ and let $\partial$ be the inner derivation of $R$ determined by the element

$$
\left(\begin{array}{llll}
0 & 1 & 0 & 0 \\
0 & 0 & 1 & 0 \\
0 & 0 & 0 & 1 \\
0 & 0 & 0 & 0
\end{array}\right) .
$$

Then one can see easily that $\partial^{7} R=(0), \partial^{6} R \neq 0, I$ is a right ideal with $\partial I \subseteq I, \partial^{4} I=$ (0) and $\partial^{3} I \neq(0)$. From this one can see the nilpotency of a derivation on a one-sided ideal in a prime ring (or even a simple Artinian ring) need not be odd, and Proposition 2 concerning the nilpotency of $\partial$ on $R$ cannot be sharpened.

\section{REFERENCES}

1. L. O. Chung and J. Luh, Nilpotency of derivations, Canad. Math. Bull. (to appear).

2. Nilpotency of derivations. II (to appear).

3. L. O. Chung, A. Kovacs and J. Luh, Algebraic derivations of prime rings, preprint.

4. I. N. Herstein, Rings with involution, Univ. of Chicago Press, Chicago, Ill., 1969. 27650

Department of Mathematics, North Carolina State University, Raleigh, North Carolina 